\title{
Recombinant DNA-produced $\alpha 1$-Antitrypsin Administered by Aerosol Augments Lower Respiratory Tract Antineutrophil Elastase Defenses in Individuals with $\alpha 1$-Antitrypsin Deficiency
}

Richard C. Hubbard, Noel G. McElvaney, Stephanie E. Sellers, Jane T. Healy, Dorothy B. Czerski, and Ronald G. Crystal Pulmonary Branch, National Heart, Lung, and Blood Institute, National Institutes of Health, Bethesda, Maryland 20892

\begin{abstract}
$\alpha 1-$ Antitrypsin ( $\alpha 1 \mathrm{AT})$ deficiency is characterized by insufficient amounts of $\alpha 1 \mathrm{AT}$ to protect the lower respiratory tract from neutrophil elastase, resulting in emphysema. Yeast-produced recombinant $\alpha 1 \mathrm{AT}$ (rAAT) has normal antielastase function but is associated with high renal clearance, thus obviating chronic intravenous administration. As an alternative, we evaluated aerosol administration of rAAT to $\alpha 1 \mathrm{AT}$-deficient individuals. After aerosol administration of single doses of 10-200 mg of rAAT, epithelial lining fluid (ELF) $\alpha 1 \mathrm{AT}$ antineutrophil elastase defenses were augmented in proportion to the dose of rAAT administered. ELF $\alpha 1 \mathrm{AT}$ levels and antineutrophil elastase capacity $4 \mathrm{~h}$ after $200 \mathrm{mg}$ rAAT aerosol were increased 40-fold over preaerosol levels, and were fivefold increased over baseline at $24 \mathrm{~h}$ after aerosol administration. rAAT was detectable in serum after aerosol, indicating that the lower respiratory tract epithelium may be permeable to $\mathrm{rAAT}$, and that aerosolized rAAT is capable of gaining access to lung interstitium. No adverse clinical effects were noted. These observations demonstrate that aerosol administration of rAAT is safe and results in significant augmentation of lung antineutrophil elastase defenses, suggesting this method is a feasible approach to therapy. Because this approach is clinically unproven, further studies will be necessary to establish the longterm clinical efficacy of aerosol therapy in $\alpha 1 \mathrm{AT}$ deficiency.
\end{abstract}

\section{Introduction}

Alpha 1-antitrypsin $(\alpha 1 \mathrm{AT})^{1}$ deficiency is a genetic disorder characterized by low plasma and lung levels of $\alpha 1 \mathrm{AT}$ and the development of emphysema by the third to fourth decades ( 1 , 2). The deficiency of $\alpha 1 \mathrm{AT}$ in the lower respiratory tract is central to the pathogenesis of the emphysema due to the critical role of $\alpha 1 \mathrm{AT}$ in protecting the alveolar structures against neutrophil elastase, a broad-spectrum protease capable of de-

Address reprint requests to Dr. Crystal, Pulmonary Branch, Building 10, Room 6D03, National Institutes of Health, Bethesda, MD 20892. Received for publication 21 February 1989 and in revised form 26 June 1989.

1. Abbreviations used in this paper: $\alpha 1 \mathrm{AT}$, alpha 1-antitrypsin; ELF, epithelial lining fluid; rAAT, recombinant $\alpha 1 \mathrm{AT}$.

The Journal of Clinical Investigation, Inc.

Volume 84, October 1989, 1349-1354 grading all major protein components of the alveolar interstitium (1-3). As a result of the low lung levels of $\alpha 1 \mathrm{AT}$, neutrophil elastase released from neutrophils in the lower respiratory tract is free to attack alveolar walls, resulting in the gradual destruction of lung parenchyma and the eventual development of emphysema (1-4). These biologic abnormalities in the lower respiratory tract can be reversed with $\alpha$ 1 AT augmentation therapy, in which human plasma-purified $\alpha 1 \mathrm{AT}$ is administered intravenously on a weekly $(2,5)$ or monthly $(6)$ basis, thus reestablishing the antineutrophil elastase protective screen of the lower respiratory tract $(2,5,6)$.

An alternative approach to therapy of $\alpha 1 \mathrm{AT}$ deficiency is to use recombinant DNA-produced $\alpha 1 \mathrm{AT}$ (rAAT) to augment the antineutrophil elastase screen of the alveolar structures. This approach has been made feasible by the demonstration that rAAT produced in yeast under the direction of a normal human $\alpha$ IAT cDNA functions as an inhibitor of neutrophil elastase in a fashion identical to that of the natural human $\alpha 1 \mathrm{AT}(7,8)$. However, unlike human plasma $\alpha 1 \mathrm{AT}$, rAAT lacks carbohydrate side chains $(7,8)$. As a result, when rAAT is administered intravenously it has a much shorter plasma halflife than does natural $\alpha 1 \mathrm{AT}$ (7). In part, this occurs because a large amount of the administered rAAT is cleared by the kidney, whereas no natural $\alpha 1 \mathrm{AT}$ appears in the urine after intravenous administration $(6,7)$. Compared with human plasma $\alpha 1 \mathrm{AT}$, intravenous administration of rAAT thus would require far more $\alpha 1 \mathrm{AT}$ to raise serum levels sufficient to protect the lung, and the large bulk of protein passing through the kidney may lead to renal disease, thus obviating this route of administration.

It may be possible, however, for rAAT to be used therapeutically to augment lung antielastase defenses if it were targeted directly to the lower respiratory tract, i.e., given by aerosol. In this regard, studies in experimental animals have demonstrated that aerosol administration permits the delivery of intact, functional rAAT to the lower respiratory tract, and that the rAAT diffuses into the alveolar interstitium, the site that must be protected in $\alpha 1 \mathrm{AT}$ deficiency (8). With this background, this study was designed to evaluate the feasibility of administering rAAT via aerosol to humans with $\alpha 1 \mathrm{AT}$ deficiency.

For aerosolization of rAAT to be considered as a form of therapy for $\alpha 1 \mathrm{AT}$ deficiency several criteria must be met, including $(a)$ the rAAT must be delivered to the lower respiratory tract of $\alpha 1 \mathrm{AT}$-deficient individuals as an intact, functional protein; (b) it must be possible to deliver an aerosol dose of rAAT that results in significant augmentation of the antineutrophil elastase defenses of the lower respiratory tract in 
$\alpha 1$ AT-deficient individuals; (c) rAAT must remain active as an inhibitor of elastase long enough for intermittent aerosol administration to be practical; $(d)$ rAAT must be able to pass through the alveolar tissues, thus affording protection to the interstitial compartment; and $(e)$ it must be safe. In this study, we demonstrate that these criteria can be met, and thus it is therefore reasonable to consider aerosol administration of rAAT as a form of therapy for $\alpha 1 \mathrm{AT}$ deficiency.

\section{Methods}

Recombinant $\alpha 1 A T$ preparation. The rAAT used in this study, supplied by Cooper Biochemicals (Mountain View, CA) and Ciba-Geigy Corp. (Summit, NJ), was produced by yeast transformed with an expressing plasmid containing a human cDNA encoding the mature normal M1 $\left(\mathrm{Val}^{213}\right)$ human $\alpha 1 \mathrm{AT}$ protein $(7,8)$. This rAAT preparation has a molecular mass of $45 \mathrm{kD}$, is identical to human plasma $\alpha 1 \mathrm{AT}$ except for an additional $\mathrm{NH}_{2}$-terminal methionine and the lack of the three carbohydrate side chains in plasma $\alpha 1 \mathrm{AT}$, and functions in an identical manner as human plasma $\alpha 1 \mathrm{AT}$ as an inhibitor of neutrophil elastase $(7,8)$.

Aerosol-generating system. rAAT was aerosolized using a compressed air driven nebulizer (Mallinckrodt) selected on the basis of its ability to generate an aerosol with droplets of optimal size $(<3 \mu \mathrm{m}$ aerodynamic diameter) for deposition in the lower respiratory tract (8, 9). rAAT aerosolized in this fashion retains its structure and function as an inhibitor of neutrophil elastase (8).

Evaluation of the ability of the aerosol device to deliver aerosol to the alveolar regions was estimated to be in the range of $10-20 \%$, estimated by quantitating the proportion of a starting dose of technetium $^{-99}$-labeled diethylenetriamine pentacetic acid impacting in the lower respiratory tract after nebulization to $\alpha 1 \mathrm{AT}$-deficient individuals.

Study population. Six $\mathrm{Z}$ homozygotes were entered into a preliminary study to evaluate the potential antigenicity of the rAAT (see below). These individuals (five males, one female, average age $46 \pm 11$ yr) were typical of $\alpha 1 \mathrm{AT}$ deficiency with emphysema and $\alpha 1 \mathrm{AT}$ serum levels of $4.3 \pm 0.4 \mu \mathrm{M}$ (all $\alpha 1 \mathrm{AT}$ levels are presented in micromolar units using a true laboratory $\alpha 1 \mathrm{AT}$ standard) (2). All data are presented as mean \pm SEM and all statistical comparisons were made using the two-tailed $t$ test.

The study population used for administration of the aerosol of rAAT consisted of $16 \alpha 1 \mathrm{AT}$-deficient individuals ( $10 \mathrm{men}, 6$ women; $39 \pm 2 \mathrm{yr}$ ). There were $14 \mathrm{Z}$ homozygotes $(\alpha 1 \mathrm{AT}$ serum levels $4.5 \pm 0.5$ $\mu \mathrm{M})$ and 2 Null $_{\text {granite falls }}$ homozygotes ( $\alpha 1 \mathrm{AT}$ levels 0 ), verified by measurement of serum $\alpha 1 \mathrm{AT}$ levels, isoelectric focusing of serum, family studies, and genotyping using specific oligonucleotide probes $(10,11) .5$ individuals were lifelong nonsmokers, 10 were exsmokers (11 \pm 2 pack-yr), and 1 was a present smoker (13 pack-yr). All had evidence of mild-to-moderate emphysema, based on physical examination, chest $x$ ray, xenon- 133 ventilation scans, technetium- $99 \mathrm{~m}$-labeled macroaggregated albumin scans and lung function (vital capacity $100 \pm 15 \%$ predicted, total lung capacity $111 \pm 12 \%$ predicted, forced expiratory volume in $1 \mathrm{~s}$ [FEV-1] $78 \pm 28 \%$ predicted, and diffusing capacity for carbon monoxide $86 \pm 25 \%$ predicted [corrected for hemoglobin and volume]) (12). Resting arterial blood gases showed $\mathrm{P}_{\mathrm{O}_{2}}$ $87 \pm 12$ Torr, $\mathrm{P}_{\mathrm{CO}_{2}} 37 \pm 3$ Torr, and $\mathrm{pH} 7.40 \pm 0.03$. Individuals with severe emphysema were excluded from the study because of the inability to use bronchoalveolar lavage to study the effect of aerosol.

Study design. Before administering rAAT by aerosol, a preliminary investigation was carried out to determine the safety of administration of rAAT to $\alpha 1 \mathrm{AT}$-deficient individuals. Over a 6-mo period, rAAT was administered subcutaneously to six $Z$ homozygotes. Three individuals received $1 \mathrm{mg}$ and three individuals received $10 \mathrm{mg}$ at biweekly intervals over a 10-wk period. Immunologic assessment included skin prick and intradermal tests to detect immediate or delayed hypersensitivity reactions, blood lymphocyte blastogenesis to yeast protein extract or to
rAAT, measurement of specific antiyeast, anti-rAAT, and antihuman $\alpha 1 \mathrm{AT}$ antibodies in serum, and quantification of serum $\alpha 1 \mathrm{AT}$ levels. There were biweekly assessments of serum chemistries, complete blood counts, erythrocyte sedimentation rate, coagulation profile, urinalysis, creatinine clearance, chest $\mathrm{x}$ rays, and lung function studies.

The aerosol study was designed to evaluate the biologic parameters relevant to the rAAT after a single-dose aerosol administration of rAAT. The study was done in two phases. First, escalating single doses of rAAT were administered by aerosol to $\alpha 1 \mathrm{AT}$-deficient individuals (10 mg, $n=2 ; 50 \mathrm{mg}, n=2 ; 100 \mathrm{mg}, n=5$; and $200 \mathrm{mg}, n=4$ ). Second, an additional three individuals were evaluated at the $200-\mathrm{mg}$ dose to determine the time dependency of biologic parameters in the lower respiratory tract modified by aerosol. For both phases of the study, before aerosol administration of rAAT, a baseline bronchoalveolar lavage was performed (2) to determine epithelial lining fluid (ELF) levels of $\alpha 1 \mathrm{AT}$ and antineutrophil elastase capacity. The lavage procedure was repeated 4 or $24 \mathrm{~h}$ after aerosol to assess the effect of rAAT aerosol administration of ELF $\alpha 1 \mathrm{AT}$ levels and antineutrophil elastase capacity.

To evaluate whether rAAT administered by aerosol diffused across the lower respiratory tract and reached the blood, we capitalized on the knowledge that the two Null $\mathrm{granite}_{\text {falls }}$ homozygotes had no $\alpha 1 \mathrm{AT}$ in the serum (13), and thus any $\alpha 1 \mathrm{AT}$ that was detectable must have come from the aerosolized rAAT. The Null granite falls $_{\text {individuals were treated }}$ with a single dose of aerosolized rAAT and serum samples were taken at $4,8,24$ and $48 \mathrm{~h}$ after the aerosol and evaluated for $\alpha 1 \mathrm{AT}$ by ELISA (2).

Serum was obtained preaerosol, 2 and $4 \mathrm{wk}$ after aerosol administration for measurement of antibodies against rAAT, yeast, and plasma $\alpha 1 \mathrm{AT}$. Subcutaneous and intradermal skin testing for hypersensitivity to rAAT or yeast proteins was performed preaerosol and 4 wk after aerosol administration. At multiple times in the $48 \mathrm{~h}$ after each aerosol administration, serum chemistries, complete blood counts, and clotting studies were evaluated. The status of the lungs was evaluated by chest $\mathrm{x}$ ray, pulmonary function tests, and arterial blood gases, obtained preaerosol, $4 \mathrm{~h}, 1 \mathrm{~d}$, and $1 \mathrm{mo}$ after aerosol administration.

To evaluate epithelial lining fluid for the presence and form of rAAT after aerosol administration, Western blot analysis of 50-fold concentrated bronchoalveolar lavage fluid was carried out by lyophilizing the fluid, reconstituting with $\mathrm{H}_{2} \mathrm{O}$, and dialyzing against phosphate buffered saline, pH 7.5. Samples were evaluated by $7.5 \%$ SDSPAGE and Western analysis with a polyclonal anti- $\alpha 1 \mathrm{AT}$ antibody (8).

Measurement of ELF $\alpha 1 \mathrm{AT}$ levels was done in quadruplicate by ELISA (2) and referenced to the volume of ELF as determined by the urea method (14). To verify that the $\alpha 1 \mathrm{AT}$ measured by ELISA represented intact rAAT, Superose-12 molecular sieve chromatographic analysis of concentrated lavage fluid of a $Z$ homozygote was carried out after aerosolization of $200 \mathrm{mg}$ rAAT. The $1-\mathrm{ml}$ fractions of the $100-\mathrm{ml}$ column eluate were individually assayed for $\alpha 1 \mathrm{AT}$ by ELISA.

Determination of the antineutrophil elastase capacity of ELF was performed as previously described (2) using a titrated neutrophil elastase standard. To demonstrate the ability of rAAT present in ELF after aerosol to combine with neutrophil elastase, samples of ELF derived from lyophilized bronchoalveolar lavage fluid from a Null granite falls $_{\text {ho- }}$ mozygote were incubated $\left(5 \mathrm{~min}, 25^{\circ} \mathrm{C}\right)$ with human neutrophil elastase $(30 \mu \mathrm{M})$. The samples were then applied to a $7.5 \%$ SDS polyacrylamide gel and transferred to nitrocellulose for Western blotting.

\section{Results}

Initial safety assessment. The 6-mo skin-testing evaluation of rAAT suggested that the rAAT would not be recognized by the immune system as foreign. Despite biweekly "immunization" with rAAT, no abnormal clinical or immunologic parameters were observed, leading to the conclusion that it was safe to proceed with aerosolization of rAAT. 
Dose dependency. Administration of varying amounts of rAAT to $\alpha 1 \mathrm{AT}$-deficient individuals resulted in a dose-response relationship for both the quantity of $\alpha 1 \mathrm{AT}$ present in the lower respiratory tract and the neutrophil elastase inhibitory capacity of ELF. Aerosol administration of single doses of $10,50,100$, or $200 \mathrm{mg}$ rAAT resulted in progressive augmentation of total ELF $\alpha 1 \mathrm{AT}$ levels (preaerosol compared with the 24-h postaerosol level after administration of $200 \mathrm{mg}$ rAAT, $P$ $<0.01$ ) (Fig. $1 A$ ). Not only were total ELF $\alpha 1$ AT levels increased, but the ability of the ELF to inhibit neutrophil elastase was also increased after rAAT aerosol (preaerosol compared with $24 \mathrm{~h}$ after aerosol administration of $200 \mathrm{mg}$ rAAT, $P<0.01$ ) (Fig. $1 B$ ). Expression of $\alpha 1$ AT levels and antineutrophil elastase capacity relative to the concentration of albumin in lavage fluid yielded the same results: preaerosol, $2.6 \pm 1.1 \mu \mathrm{g} \alpha 1 \mathrm{AT} / \mu \mathrm{g}$ albumin; after $10 \mathrm{mg}$ aerosol, $2.5 \pm 2.4 \mathrm{ng}$ $\alpha 1 \mathrm{AT} / \mu \mathrm{g}$ albumin; after $100 \mathrm{mg}$ aerosol, $7.2 \pm 2.5 \mathrm{ng} \alpha 1 \mathrm{AT} / \mu \mathrm{g}$ albumin; after $200 \mathrm{mg}$ aerosol, $13.2 \pm 3.0 \mathrm{ng} \alpha 1 \mathrm{AT} / \mu \mathrm{g}$ albumin. Bronchoalveolar lavage fluid antineutrophil elastase capacity per microgram albumin yielded similar results (data not shown). When evaluated 1 mo later, for all individuals, there was a complete reversion to preaerosol levels of $\alpha 1 \mathrm{AT}$ and antineutrophil elastase capacity $(P>0.5$, all comparisons).

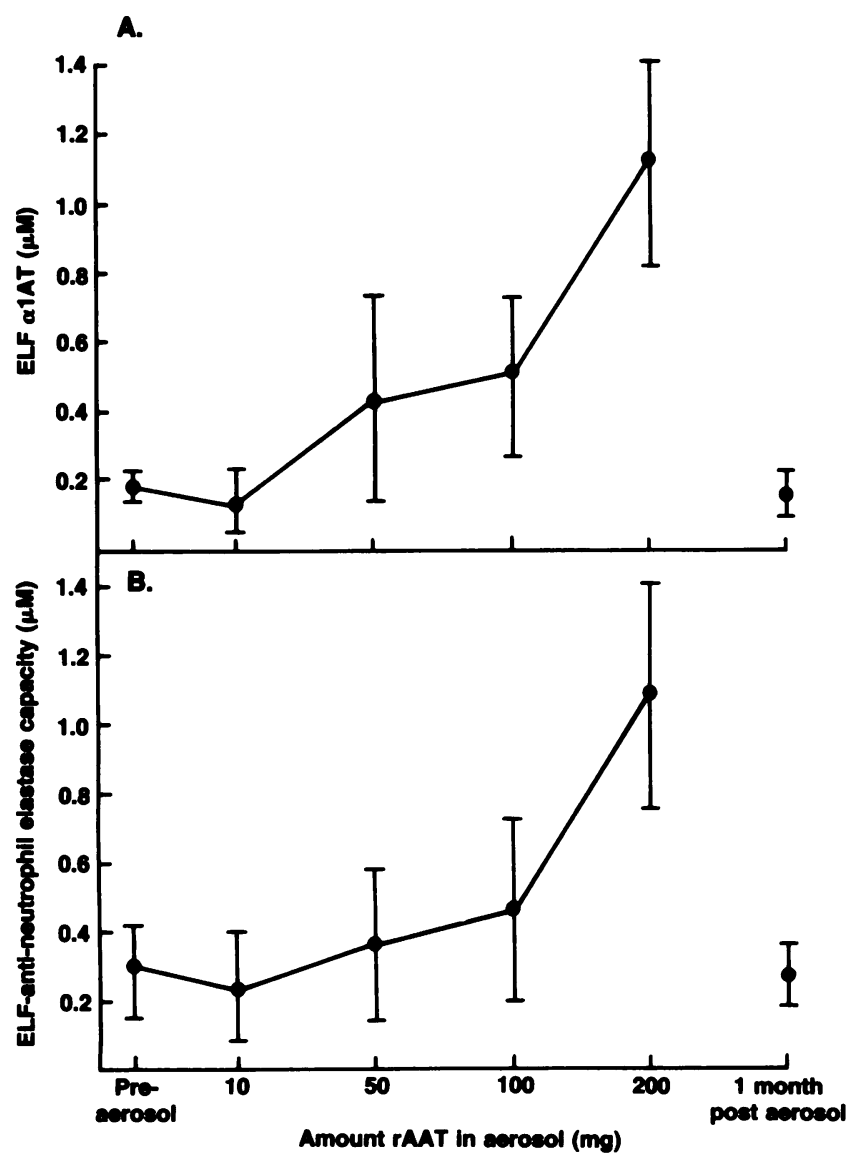

Figure 1. Effect of increasing doses of aerosolized rAAT on ELF $\alpha 1 \mathrm{AT}$ levels and ELF antineutrophil elastase (NE) capacity of $Z$ homozygous individuals. Lavage was performed before or $24 \mathrm{~h}$ and 1 mo after aerosol of rAAT. $(A)$ Total $\alpha 1 \mathrm{AT}$ levels in lavage fluid measured by ELISAs. (B) Anti-NE capacity determined by the ability lavage fluid to inhibit a fixed amount of human neutrophil elastase. Concentrations are expressed relative to the volume of ELF recovered.
Structure of the rAAT delivered by aerosol. Analysis of lavage fluid demonstrated that the rAAT delivered by aerosol to the lower respiratory tract remained intact during the delivery process. As demonstrated by Western blot analysis of concentrated lavage fluid obtained from a $\mathrm{Z}$ homozygous individual $24 \mathrm{~h}$ after aerosol administration of $200 \mathrm{mg}$ rAAT, administration of rAAT via the aerosol route resulted in clearly detectable amounts of intact rAAT in lower respiratory tract ELF (Fig. 2). Using SDS acrylamide electrophoresis and a polyclonal antibody that recognizes both human plasma $\alpha 1 \mathrm{AT}$ and rAAT, plasma $\alpha 1 \mathrm{AT}$ with its molecular mass of $52 \mathrm{kD}$ (lane 1 ), is distinguishable by the difference in mass from rAAT, with a molecular mass of $45 \mathrm{kD}$ (lane 2). Before aerosol administration of rAAT, the ELF contained only the expected endogenous $52 \mathrm{kD} \alpha 1 \mathrm{AT}$ (lane 3 ). In contrast, after aerosol administration of rAAT, ELF contained both the 52-kD endogenous plasma $\alpha 1 \mathrm{AT}$ and the $45-\mathrm{kD}$ rAAT (lane 4 ). The rAAT present in the ELF was intact, and looked identical to the rAAT standard that had not been aerosolized, i.e., the protein did not undergo fragmentation or was otherwise reduced in size either during aerosol or once deposited in the lung. Furthermore, consistent with the ELISA data, comparison of the relative intensity of the endogenous $\alpha 1 \mathrm{AT}$ and rAAT on the SDS gels suggested that even $24 \mathrm{~h}$ after delivery, $200 \mathrm{mg}$ aerosol administration of rAAT provides more $\alpha 1 \mathrm{AT}$ to the epithelial surface than does the endogenous $\alpha 1 \mathrm{AT}$ of the $\alpha 1 \mathrm{AT}$ deficient individual. Additional evidence that recovered rAAT was not fragmented was provided by chromatography of the concentrated lavage fluid; evaluation of all fractions by $\alpha 1 \mathrm{AT}$-specific ELISA demonstrated all $\alpha 1 \mathrm{AT}$ (endogenous plus rAAT) in the 42-55-kD range.

Time dependency. Evaluation of ELF levels of $\alpha 1 \mathrm{AT}$ at different times after a single aerosol dose of rAAT demonstrated a time dependent change in the amount of $\alpha 1 \mathrm{AT}$ in ELF (Fig. 3). Analysis of lavage fluid obtained $4 \mathrm{~h}$ after $200 \mathrm{mg}$ rAAT aerosol showed that the ELF levels of $\alpha 1 \mathrm{AT}$ were elevated $>40$-fold over preaerosol ELF $\alpha 1$ AT levels $(P<0.01)$. By $24 \mathrm{~h}$, the ELF $\alpha 1 \mathrm{AT}$ levels had fallen, but were still fivefold over preaerosol levels $(P<0.01)$. When viewed in the context of the ELF levels of $\alpha 1 \mathrm{AT}$ in normals, single-dose administration of $200 \mathrm{mg}$ rAAT normalized ELF $\alpha 1 \mathrm{AT}$ levels in $\alpha 1 \mathrm{AT}$ -

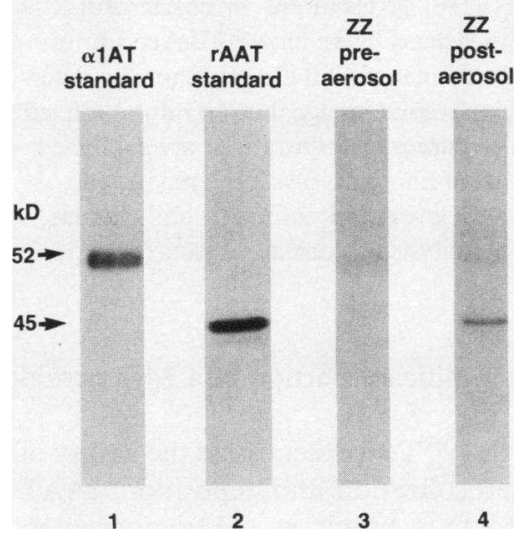

Figure 2. Evaluation of the structure of (rAAT) in lavage fluid of $a$ homozygous individual after the aerosol administration of rAAT. Shown is a Western blot of a SDS-PAGE of purified $\alpha 1 \mathrm{AT}$ standards and of concentrated lavage fluid before and 24 hr after administration of $200 \mathrm{mg}$ rAAT. Lane 1 , human plasma purified $\alpha 1$ AT standard; lane 2, rAAT standard; lane 3, lavage fluid from a $\mathrm{Z}$ homozygous individual before aerosol administration of rAAT; lane 4, lavage fluid from the same $Z$ homozygous individual $24 \mathrm{~h}$ after the aerosol administration of $200 \mathrm{mg}$ of rAAT. The relative molecular masses of each type of $\alpha 1 \mathrm{AT}$ are indicated (arrows). 


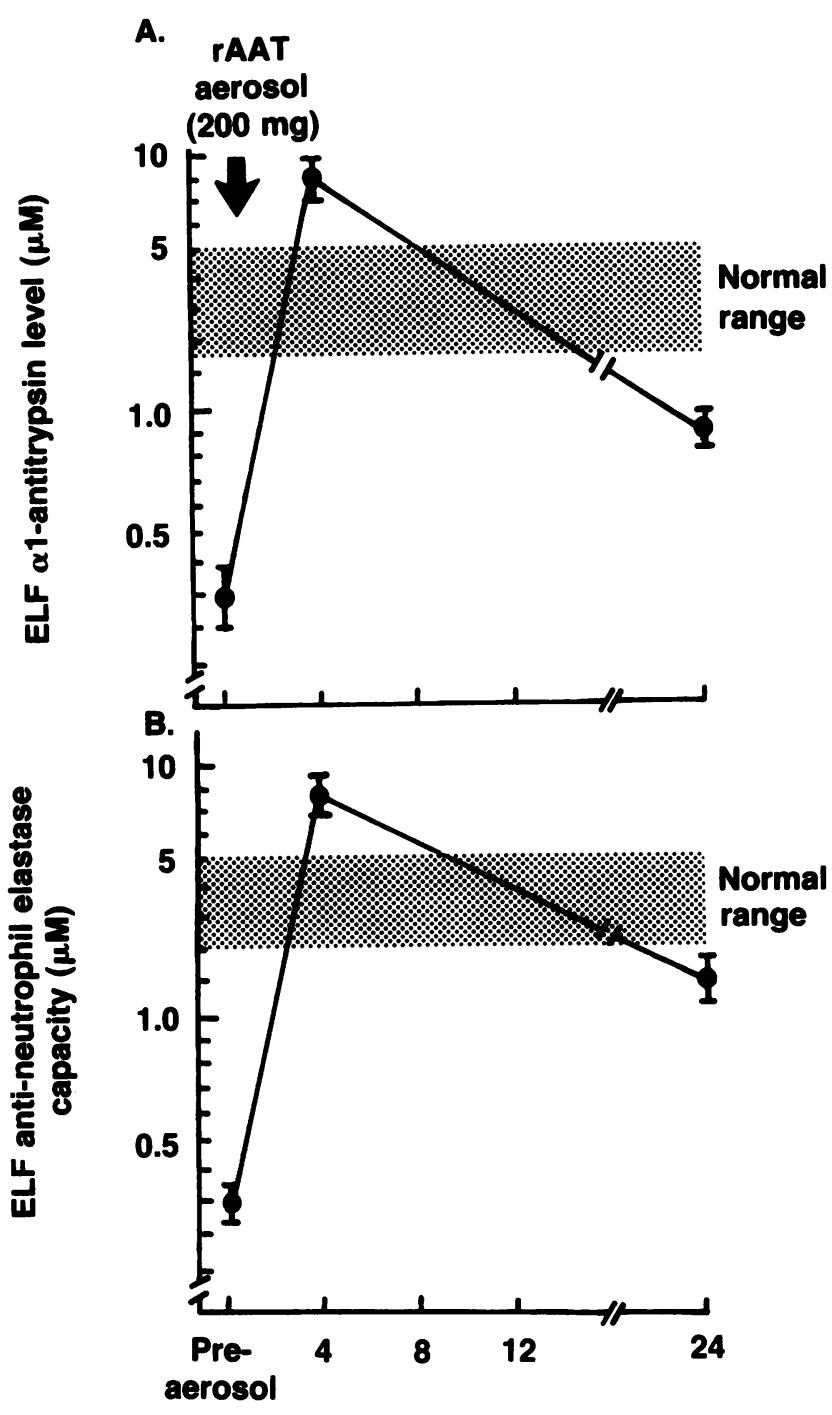

Time after aerosol (h)

Figure 3. Time course of the augmentation of ELF $\alpha 1 \mathrm{AT}$ levels and ELF anti-neutrophil elastase capacity following aerosol administration of rAAT. rAAT $(200 \mathrm{mg})$ was administered by aerosol to seven $\alpha 1 \mathrm{AT}$ deficient individuals and bronchoalveolar lavage performed at 4 or $24 \mathrm{~h}$ later. $(A)$ Total ELF $\alpha 1 \mathrm{AT}$ levels in pre- or postaerosol bronchoalveolar lavage fluid measured by an enzyme linked immunoassay specific for $\alpha 1 \mathrm{AT}$. (B) Antineutrophil elastase capacity determined by the ability of bronchoalveolar lavage fluid to inhibit a fixed amount of human neutrophil elastase. Concentrations are expressed (log scale) relative to the epithelial lining fluid volume recovered. The range for normal ELF $\alpha 1 \mathrm{AT}$ levels and antineutrophil elastase capacity was determined from analysis of normal subjects (21).

deficient individuals for a significant portion of a $24-\mathrm{h}$ period after aerosol.

Function of recovered $r A A T$. To further assess the ability of rAAT to function after aerosolization and deposition, rAAT was administered by aerosol to two Null granite falls homozygotes, thus permitting assessment of the nature of the interaction between rAAT and neutrophil elastase in the absence of the confounding effects of endogenous $\alpha 1 \mathrm{AT}$. As expected, ELF of a Null granite falls homozygote contained no $\alpha 1 \mathrm{AT}$ (Fig. 4, lane 1). The addition in vitro of rAAT to Null granite falls ELF demon-

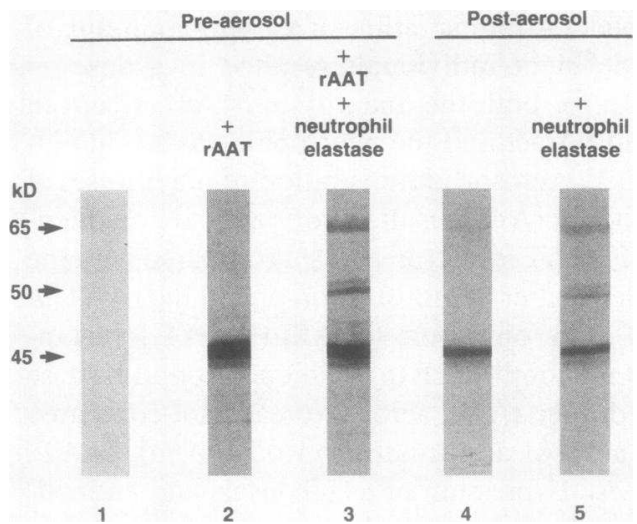

Figure 4. Demonstration that aerosolized rAAT remains intact and functional as an inhibitor of neutrophil elastase (NE) after in vivo administration. Shown is a Western blot analysis of lavage fluid from a homozygous Null granite falls $_{\text {individual obtained before and after }}$ aerosol administration of rAAT. Lane 1, lavage fluid before rAAT aerosol; lane 2, lavage fluid plus rAAT added in vitro to the lavage fluid; lane 3, lavage fluid plus rAAT and NE added to the lavage fluid in vitro; lane 4, lavage fluid obtained $4 \mathrm{~h}$ after aerosol administration of $200 \mathrm{mg}$ rAAT; lane 5 , lavage fluid obtained $4 \mathrm{~h}$ after aerosol administration of $200 \mathrm{mg}$ rAAT $+\mathrm{NE}$ added in vitro. Arrows indicate relative molecular masses.

strated the expected $45-\mathrm{kDa}$ protein band corresponding to intact rAAT standard (lane 2). When rAAT and neutrophil

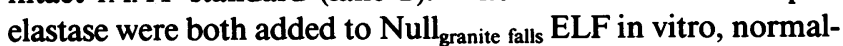
sized rAAT $(45 \mathrm{kD})$ as well as rAAT complexed with neutrophil elastase $(66 \mathrm{kD}$, the complete rAAT complexed with neutrophil elastase, and to a lesser extent, $50 \mathrm{kD}$, the rAAT-neutrophil elastase complex in which the rAAT has lost the fragment $\mathrm{COOH}$-terminal to the active site met $^{358}$; see reference 15 for details) were observed (lane 3). Strikingly, analysis

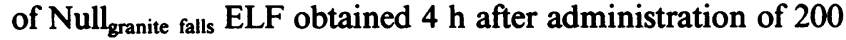
mg rAAT demonstrated the presence of both uncomplexed rAAT (45 kD) and rAAT at a higher molecular mass (66 kD), suggesting but not proving, that the rAAT delivered to the lower respiratory tract by aerosol may have combined with neutrophil elastase in vivo (lane 4). Moreover, consistent with the observed enhancement of ELF antineutrophil elastase capacity by the aerosol administration of rAAT, the further addition of neutrophil elastase in vitro demonstrated that the uncomplexed $45 \mathrm{kD}$ rAAT present in postaerosol ELF was still functionally active and retained its ability to combine with and form the $66-\mathrm{kD}$ (and to a lesser extent the 50-kD) complex of rAAT-neutrophil elastase (lane 5).

Permeability of the lower respiratory tract to aerosolized $r A A T$. To determine if the lung epithelial surface is permeable to aerosolized rAAT, the serum of two homozygous Null $_{\text {granite falls }}$ individuals was evaluated for the presence of $\alpha 1 \mathrm{AT}$ before, and at intervals over a 48-h period after aerosol administration of $200 \mathrm{mg}$ rAAT. By definition, consistent with the null status of the study individuals, no $\alpha 1 \mathrm{AT}$ was present in serum preaerosol. However, after aerosol administration, rAAT was detectable in serum in one individual at $8 \mathrm{~h}$ after aerosolization and in both patients at $24 \mathrm{~h}$ after aerosol (Fig. 5). By $48 \mathrm{~h}, \mathrm{rAAT}$ was not detectable in the serum of either individual. rAAT that reached the lung epithelial surface via aerosol thus was able to pass across the lower respiratory tract 


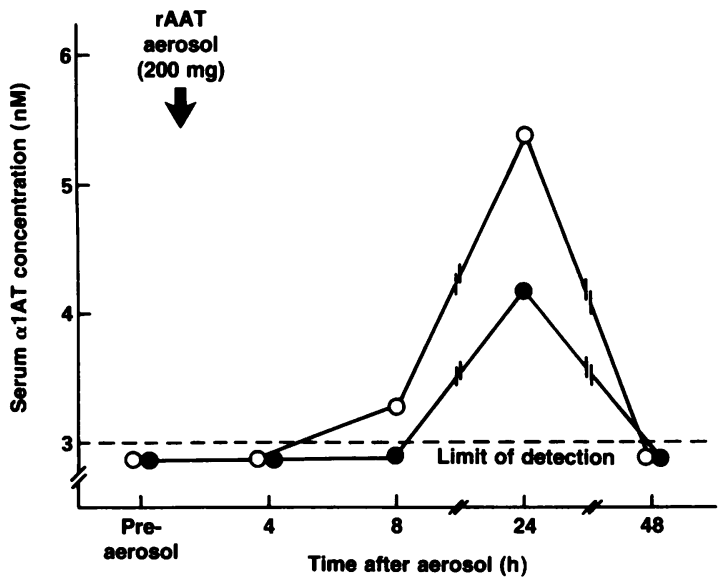

Figure 5. Serum $\alpha 1 \mathrm{AT}$ levels in two homozygous Null granite falls individuals before and after aerosol administration of rAAT. rAAT $(200 \mathrm{mg}$ ) was administered by aerosol and serum was obtained before aerosol and at intervals after aerosol and assayed for $\alpha 1 \mathrm{AT}$ by ELISA. The limit of sensitivity of the immunoassay for $\alpha 1 \mathrm{AT}$ in serum is $3 \mathrm{nM}$. Each individual is represented by a separate symbol $(\circ, \bullet)$.

epithelium and enter the circulation, i.e., the lower respiratory tract epithelium is permeable to rAAT deposited on the alveolar surface by aerosol, suggesting at least some of the rAAT must have reached the alveolar interstitial tissues.

Safety. No adverse reaction was noted among any of the patients receiving rAAT by aerosol. Each aerosol administration was well tolerated and no immediate or late symptoms were noted. No clinical laboratory parameters were significantly changed at 4,24 , or 48 h or 1 mo after aerosol, when compared with the preaerosol values (data not shown). Furthermore, there was no evidence of inflammation developing in the lower respiratory tract as assessed by comparison of the bronchoalveolar lavage fluid cellular constituents of pre- and postaerosol lavage fluid; preaerosol, $21 \pm 14 \times 10^{6}$ total cells recovered, $83 \pm 10 \%$ macrophages, $12 \pm 9 \%$ lymphocytes, $4 \pm 4 \%$ neutrophils; postaerosol, $16 \pm 13 \times 10^{6}$ total cells, $85 \pm 16 \%$ macrophages, $9 \pm 9 \%$ lymphocytes, $5 \pm 4 \%$ neutrophils $(P>0.5$ pre- vs. postaerosol, all comparisons). Importantly, aerosol administration of rAAT did not result in development of immunologic sensitization against rAAT. In this regard, skin testing with prick and subcutaneous injection of rAAT, yeast extract, and human $\alpha 1 \mathrm{AT}$ did not demonstrate a positive reaction in any individual before or after aerosol administration of rAAT and no study individual developed serum antibodies to rAAT, yeast extract, or human $\alpha 1 \mathrm{AT}$.

\section{Discussion}

This study demonstrates that aerosol administration of rAAT is a feasible and effective method of augmenting the antielastase defenses of the lower respiratory tract in $\alpha 1 \mathrm{AT}$ deficiency. By using an appropriately designed aerosol-generating system, significant quantities of intact, functional rAAT can be deposited directly on the alveolar epithelial surface, resulting not only in increased $\alpha 1 \mathrm{AT}$ levels and antineutrophil elastase capacity in ELF, but also, due to the permeability of the alveolar epithelium to rAAT, increased levels of rAAT in lung interstitial tissues as well. Administration of rAAT results in dose- and time-dependent changes in ELF $\alpha 1 \mathrm{AT}$ and antineutrophil elastase levels in a manner that suggests that once or twice daily aerosol administration of rAAT should result in sustained levels of antielastase protection to the lower respiratory tract. When interpreted in view of the safety of its administration, as evidenced by the lack of pulmonary, immunologic, or general clinical adverse effect of either single-dose or chronic administration of rAAT, aerosol rAAT administration may constitute a reasonable approach to therapy for $\alpha 1 \mathrm{AT}$ deficiency.

Because the lung has only a limited capacity to produce its own antineutrophil elastase protective screen, the lower respiratory tract is dependent on antielastases produced outside of the lung, principally $\alpha 1 \mathrm{AT}(3,16)$. The vast majority of $\alpha 1 \mathrm{AT}$ is produced in the liver by hepatocytes and reaches the lung via the circulation; in the normal individual, sufficient $\alpha 1 \mathrm{AT}$ diffuses from plasma into the lung to inhibit the burden of elastase to which the lung is exposed $(1,3,5,11)$. This same concept of augmenting lower respiratory tract antielastase defenses is used in intravenous augmentation therapy for $\alpha 1 \mathrm{AT}$ deficiency, in which plasma purified $\alpha 1 \mathrm{AT}$ is administered intravenously and passively reaches the lung via diffusion from the circulation $(2,5,6)$. Using doses of $60 \mathrm{mg} / \mathrm{kg}$ per wk, intravenous therapy delivers sufficient $\alpha 1 \mathrm{AT}$ to the lungs to reverse the imbalance between elastase and antielastase at the alveolar surface, where the concentration of $\alpha$ IAT in ELF is $\sim 10 \%$ the concentration in plasma (2). In reaching the alveolar epithelial surface, the infused $\alpha 1 \mathrm{AT}$ passes through capillary endothelium, lung interstitium, and finally the alveolar epithelial membrane, in the process providing elastase protection throughout the lung, including the lung interstitium, the critical site requiring protection from elastolytic degradation.

In contrast, aerosol administration uses the opposite approach to augmenting lung antielastase defenses. Once the aerosolized rAAT passes the upper airway anatomic barriers, the first lower respiratory tract tissue reached by aerosolized rAAT is the alveolar epithelium (8). After alveolar deposition, rAAT must traverse through lung tissues in the reverse order traveled by intravenously administered $\alpha 1 \mathrm{AT}$, moving first through the alveolar epithelium, lung interstitium, and capillary endothelium, ultimately reaching the general circulation. Aerosol administration represents a potentially far more efficient method of delivering rAAT to the alveolar surface than intravenous therapy. In this regard, $4 \mathrm{~h}$ after the $200-\mathrm{mg}$ aerosol dose, the ELF $\alpha 1 \mathrm{AT}$ level was $\sim 9 \mu \mathrm{M}$. It has been estimated that the total lung ELF volume is $\sim 100 \mathrm{ml}$, so that a total of $40 \mathrm{mg} \mathrm{rAAT}$, or $20 \%$ of the initial dose, is present in ELF. This compares with an efficiency rate of only $2 \%$ for the delivery of infused $\alpha$ 1AT to the lower respiratory tract (2).

A major question is how effectively aerosol administration provides antielastase protection to the alveolar interstitium, particularly in view of the relatively high degree of resistance to diffusion of macromolecules presented by the alveolar epithelium compared with the endothelial membrane $(17,18)$. However, several lines of evidence suggest that the aerosolized rAAT is not restricted to the alveolar surface, that it does move from the epithelial surface into lung interstitial tissues. First, as was demonstrated in this study, rAAT gains access to the circulation in $\alpha 1 \mathrm{AT}$ deficient individuals after aerosol administration, thus implying that it passed from the alveolar epithelial surface into lung interstitium and then to the general circulation, either through lung interstitial lymphatic drainage, or 
directly into the circulation through alveolar capillaries. $\mathrm{Ab}$ sorption of some of the aerosolized rAAT by transport across the oropharynx or the large airways may also occur.

Second, studies in sheep, in which it is possible to directly sample lung interstitial lymph, have shown that after rAAT aerosol administration, rAAT is present in lymph draining the lung interstitium, as well as in blood plasma, with an $\sim 10$ fold difference in concentration between lung interstitial lymph and plasma, indicating that passage of rAAT into lung interstitium and lymphatics does occur to a significant degree (8). Although rAAT and plasma $\alpha 1 \mathrm{AT}$ may have different pharmacokinetics in the lung because of their different structure, these observations are also consistent with studies of aerosolization of plasma $\alpha$ IAT to dogs, showing alveolar deposition and retention for as long as $6 \mathrm{~h}$ after aerosol (19), and aerosolization to sheep demonstrating passage of plasma $\alpha 1 \mathrm{AT}$ into the pulmonary interstitium (20). Although it is not possible to quantify the amounts of rAAT reaching lung interstitium after aerosol to humans, nor is it known what levels of $\alpha 1 \mathrm{AT}$ are present in the interstitium of normal individuals, it is nonetheless clear that the aerosol route does result in delivery of some rAAT throughout the lower respiratory tract.

Aerosol therapy may represent a significant step in reducing the cost of chronic augmentation therapy for $\alpha 1 \mathrm{AT}$ deficiency. Although the costs of the aerosol delivery system and the drug itself cannot be accurately predicted at this time, the major savings for this form of therapy is likely to be in the amount of drug required, because the single greatest cost of augmentation therapy is the cost of the drug itself. Because for a typical $70-\mathrm{kg}$ individual the amount of drug required is reduced from $4,200 \mathrm{mg} / \mathrm{wk}$ (for intravenous therapy) to an estimated $1,400 \mathrm{mg} / \mathrm{wk}$ (for aerosol therapy), a reduction by twothirds in the amount of drug needed is possible.

In this context, this investigation is an important first step in demonstrating that aerosolization of rAAT may constitute effective therapy for $\alpha 1 \mathrm{AT}$ deficiency. With the background knowledge that it is possible to safely administer plasma $\alpha 1 \mathrm{AT}$ to humans twice daily by aerosol (20), and with the safety and biologic efficacy of this study, it is reasonable to justify a study of the chronic administration of rAAT to humans with $\alpha 1 \mathrm{AT}$ deficiency.

\section{Acknowledgments}

We are grateful to Paul Kirk, Russ Phares, Niek Roosdorp, and Mike Ullman (Cooper Laboratories, Inc., Mountain View, CA) and H.-P. Schnebli (CIBA-GEIGY) for all of their help in developing the recombinant $\alpha 1$-antitrypsin for human use; and Michael Matthay, Cardiovascular Research Institute, Department of Medicine and Physiology, University of California, San Francisco for his helpful advice.

\section{References}

1. Gadek, J. E., and R. G. Crystal. 1982. $\alpha 1$-antitrypsin deficiency. In The Metabolic Basis of Inherited Disease. J. B. Stanbury, J. B. Wyngaarden, D. S. Fredrickson, J. L. Goldstein, and M. S. Brown, editors. McGraw-Hill Book Co., New York. 1450-1467.

2. Wewers, M. D., M. A. Casolaro, S. E. Sellers, S. C. Swayze, K. M. McPhaul, J. T. Wittes, and R. G. Crystal. 1987. Replacement therapy for alpha-1-antitrypsin deficiency associated with emphysema. $N$. Engl. J. Med. 316:1055-1062.

3. Gadek, J. E., G. A. Fells, R. L. Zimmerman, S. I. Rennard, and
R. G. Crystal. 1981. Antielastases of the human alveolar structures. $J$. Clin. Invest. 68:889-898.

4. Janoff, A. 1985. Elastases and emphysema. Am. Rev. Respir. Dis. 132:417-433.

5. Hubbard, R. C., and R. G. Crystal. 1988. Alpha-1-antitrypsin augmentation therapy for alpha-1-antitrypsin deficiency. Am. J. Med. 84(Suppl. 6A):52-62.

6. Hubbard, R. C., S. Sellers, D. Czerski, L. Stephens, and R. G. Crystal. 1988. Biochemical efficacy and safety of monthly augmentation therapy for $\alpha 1$-antitrypsin deficiency. JAMA (J. Am. Med. Assoc.). 260:1259-1264.

7. Casolaro, M. A., G. Fells, M. Wewers, J. E. Pierce, F. Ogushi, R. Hubbard, S. Sellers, J. Forstrom, D. Lyons, G. Kawasaki, and R. G. Crystal. 1987. Augmentation of lung antineutrophil elastase capacity with recombinant human $\alpha$-1-antitrypsin. J. Appl. Physiol. 63:20152023.

8. Hubbard, R. C., M. A. Casolaro, M. Mitchell, S. E. Sellers, F. Arabia, M. A. Matthay, and R. G. Crystal. 1988. Fate of aerosolized recombinant DNA-produced $\alpha 1$-antitrypsin: use of the epithelial surface of the lower respiratory tract to administer proteins of therapeutic importance. Proc. Natl. Acad. Sci. USA. 86:680-684.

9. Agnew, J. E. 1984. Physical properties and mechanisms of deposition of aerosols. In Aerosols and the Lung: Clinical and Experimental Aspects. S. W. Clarke and D. Pavia, editors. Butterworths, London. 49-70.

10. Brantly, M., T. Nukiwa, and R. G. Crystal. 1988. Molecular basis of alpha-1-antitrypsin deficiency. Am. J. Med. 84:52-62.

11. Crystal, R. G., M. L. Brantly, R. C. Hubbard, D. T. Curiel, D. J. States, and M. D. Holmes. 1989. The alpha 1-antitrypsin gene and its mutations. Chest. 95:196-208.

12. Fulmer, J. D., W. C. Roberts, E. R. Von Gal, and R. G. Crystal. 1977. Small airways in idiopathic pulmonary fibrosis: comparison of morphologic and physiologic observations. J. Clin. Invest. 60:595610.

13. Nukiwa, T., H. Takahashi, M. Brantly, M. Courtney, and R. G. Crystal. 1987. $\alpha 1$-antitrypsin Null $_{\text {granite falls, }}$ a non-expressing $\alpha 1$-antitrypsin gene associated with a frameshift to stop mutation in a coding exon. J. Biol. Chem. 262:11999-12004.

14. Rennard, S. I., G. Basset, D. Lecossier, K. M. O'Connell, P. Pinkston, P. G. Martin, and R. G. Crystal. 1986. Estimation of volume of epithelial lining fluid recovered by lavage using urea as marker of dilution. J. Appl. Physiol. 60:532-538.

15. Travis, J., M. Owen, P. George, R. Carrell, S. Rosenberg, R. A. Hallewell, and P. J. Barr. 1985. Isolation and properties of recombinant DNA produced variants of human $\alpha 1$-proteinase inhibitor. $J$. Biol. Chem. 260:4384-4389.

16. Wewers, M. D., M. A. Casolaro, and R. G. Crystal. 1987. Comparison of alpha-1-antitrypsin levels and antineutrophil elastase capacity of blood and lung in a patient with the alpha-1-antitrypsin phenotype null-null before and during alpha-1-antitrypsin augmentation therapy. Am. Rev. Respir. Dis. 135:539-543.

17. Gorin, A. B., and P. A. Stewart. 1979. Differential permeability of endothelial and epithelial barriers to albumin flux. J. Appl. Physiol. 47:1315-1324.

18. Matthay, M. A., Y. Berthiaume, and N. C. Staub. 1985. Longterm clearance of liquid and protein from the lungs of unanesthetized sheep. J. Appl. Physiol. 59:928-934.

19. Smith, R. M., and R. G. Spragg. 1988. Production and administration to dogs of aerosols of alpha-1-proteinase inhibitor. Am. J. Med. 84(6A):48-52.

20. Hubbard, R. C., L. Stephens, and R. G. Crystal. 1989. Delivery of $\alpha 1$-antitrypsin by aerosol: direct augmentation of lung anti-elastase defenses in $\alpha 1$-antitrypsin deficiency. Ann. Intern. Med. 111:206-212.

21. Ogushi, F., R. C. Hubbard, G. A. Fells, M. A. Casolaro, D. T. Curiel, M. L. Brantly, and R. G. Crystal. 1987. Evaluation of the S-type of alpha-1-antitrypsin as an in vivo and in vitro inhibitor of neutrophil elastase. Am. Rev. Respir. Dis. 137:364-370. 